\title{
FIXED POINTS OF MULTIVALUED NONEXPANSIVE MAPS
}

\author{
T. HUSAIN \\ Department of Mathematics and Statistics \\ McMaster University \\ Hamilton, Ontario Canada L8S $4 \mathrm{Kl}$ \\ and \\ ABDUL LATIF \\ Department of Mathematics \\ Gomal University \\ D.I. Khan, Pakistan \\ (Received June 21, 1990 and in revised form December 12, 1990)
}

\begin{abstract}
Fixed point thecrems for multivalued contractive-type and nonexpansivetype maps on complete metric spaces and on certain closed bounded convex subsets of Banach spaces have been proved. They extend some known results due to Browder, Husain and Tarafdar, Karlovitz and Kirk.
\end{abstract}

KEY WORDS AND PHRASES: Fixed points of contractive-type and nonexpansive-type multivalued maps.

1980 AMS SUBJECT CLASSIFICATION CODE: 54H25

\section{INTRODUCTION.}

The well-known Banach fixed point theorem on complete metric spaces (specifically, each contraction self-map of a complete metric space has a unique fixed point) has been extended and generalized in different directions. For example, see Edelstein [4,5], Kasahara [10], Rakotch $[15,16]$ and others. One of its generalizations is for nonexpansive single valued maps on certain subsets of a Banach space. Indeed, these fixed points are not necessarily unique. See, for example, Browder $[1,2,3]$ and Kirk [11]. Fixed point theorems for nonexpansive multivalued maps on certain subsets of a Banach space have also been established by several authors.

Let $\mathrm{d}_{\mathrm{H}}$ denote the Hausdorff metric on the space of all bounded non-empty subsets of a metric space $(X, d)$. A multivalued map $J: X \rightarrow 2^{X}$ (the collection of all nonempty subsets of $\mathrm{X}$ ) with bounded subsets as values is called contractive if

$$
d_{H}(J(x), J(y)) \leq h d(x, y)
$$

for all $x, y$ in $X$ and for a fixed number $h, 0 \leq h<1$. If the Lipschitz constant $h=1$, then $\mathrm{J}$ is called a multivalued nonexpansive mapping. Among many authors, Nadler [13] and Lami Dozo [12] proved fixed point theorems for these maps under certain conditions. 
We note that an element $x \in X$ is called a fixed point of a multivalued map $J: X \rightarrow 2^{X}$ if $x \in \mathrm{J}(\mathrm{x})$.

Husain and Tarafdar [7] introduced the notion of a nonexpansive-type multivalued map and proved a fixed point theorem on compact intervals of the real line. A question of extending this result on certain subsets of a Banach or Hilbert space was raised.

In this paper, we deal with this question and prove fixed point theorems for nonexpansive-iype and contractive-type multivalued maps (Theorem 2.3, Theorem 3.4). One of the corollaries (Corollary 3.8) of Theorem 3.4 extends a result of Husain and Tarafdar [7]. Since it follows that each nonexpansive and each contraction map is also a non-expansivetype and contractive-type map respectively, our results include those of Browder [2], Karlovitz [9] and others, not to mention the fact that the Banach fixed point theorem is a special case of our results. Moreover, Theorem 3.4 contains the following as a special case: Each single-valued nonexpansive map on a nonempty closed, convex, bounded subset of a reflexive Banach space satisfying Opial's condition has a fixed point. This can also be derived from Kirk [11]. Lastly, we prove a common fixed point theorem for a sequence of multivalued contractive-type maps (Theorem 4.1).

\section{FIXED POINTS OF CONTRACTIVE-TYPE MULTIVALUED MAPS}

Let $M$ be a nonempty subset of a metric space $(X, d)$. The first author and Tarafdar [7] call a map $\mathrm{J}: \mathrm{M} \rightarrow 2^{\mathrm{X}}$ nonexpansive (here it is called nonexpansive-type to avoid confusion) if for all $x \in M, u_{x} \in J(x)$ there exists $v_{y} \in J(y)$ for all $y \in M$ such that

$$
d\left(u_{x}, v_{y}\right) \leq d(x, y) .
$$

Clearly, this notion generalizes the usual concept of nonexpansive [3] maps. Further, if in the above inequality we have

$$
d\left(u_{x}, v_{y}\right) \leq h d(x, y)
$$

for some fixed $h, 0 \leq h<1$, then we call it a contractive-type map. The notion of contraction for single-valued maps is clearly coincident with a contractive-type mapping. Moreover, each contractive-type mapping is a nonexpansive-type map.

We begin with some examples of nonexpansive-type and contractive-type maps.

EXAMPLES 2.1 (a) Let $\left\{\mathrm{f}_{\alpha} ; \alpha \in \mathrm{I}\right\}$ be a family of single-valued contraction mappings on a non-empty subset $M$ of a metric space $(X, d)$ into $X$, with the same Lipschitz constant $h=h_{\alpha}$ [i.e. for each $\alpha \in I, f_{\alpha}: M \rightarrow X$ and $d\left(f_{\alpha}(x), f_{\alpha}(y)\right) \leq h d(x, y)$ for all $x, y \in M, 0 \leq h<1]$. Then the multivalued mapping $J(x)=\left\{f_{\alpha}(x): \alpha \in I\right\} \quad(x \in M)$, in a contractive-type map of $M$ into $2^{X}$, with the Lipschitz constant $h$. Instead of $h_{\alpha}=h$, it is enough to assume that $0 \leq \mathrm{h}_{\alpha}=\mathrm{h}$, for all $\alpha$.

(b) Let $X=\mathbb{R}^{2}$ with the usual Euclidean metric d. For $M=\left\{x=\left(x_{1}, x_{2}\right) \in X\right.$ : $\left.x_{i} \geq 0, \quad i=1,2\right\}$, define

$$
J(x)=\left\{ \pm\left[\frac{x_{1}}{2}, \frac{x_{2}}{2}\right], \pm\left[\frac{x_{1}}{3}, \frac{x_{2}}{3}\right], \ldots\right\} \in 2^{X} \quad(x \in M) .
$$


Then $\mathrm{J}$ is a contractive-type multivalued mapping with $\mathrm{h}=\frac{1}{2}$. For if $u_{x}=$ $\left[\frac{x_{1}}{n}, \frac{x_{2}}{n}\right] \in J(x)$, where $n$ is any positive integer greater than or equal to 2 , then for any $y \in M$, put $u_{y}=\left[\frac{y_{1}}{n}, \frac{y_{2}}{n}\right] \in J(y)$. Clearly

$$
d\left(u_{x}, u_{y}\right)=\sqrt{\frac{1}{n^{2}}\left[\left(x_{1}-y_{1}\right)^{2}+\left(x_{2}-y_{2}\right)^{2}\right]} \leq \frac{1}{2} d(x, y) .
$$

This inequality is also true for any negative integer $n$ less than or equal to -2 .

(c) Let $X=\mathbb{R}$ with the usual metric $d$ and $M=[0,1]$. Consider

$$
J(x)=\left[\frac{x}{3}, \frac{x}{2}\right] \quad(x \in M)
$$

then it defines a contractive-type multivalued mapping of $M$ into $2^{X}$ with $h=\frac{1}{2}$. More generally, the multivalued mapping

$$
J(x)=\left[\frac{x}{n+1}, \frac{x}{n}\right] \quad(x \in M)
$$

is a contractive-type mapping with $\mathrm{h}=\frac{1}{\mathrm{n}}$ for any positive integer $\mathrm{n} \geq 2$.

The following example shows that there are nonexpansive-type multivalued maps which are not contractive-type

EXAMPLE 2.2 Let $M=X=\mathbb{R}$ with the usual metric d. Define

$$
J(x)=\left\{x-\tan ^{-1}(x), \frac{x}{2}-\tan ^{-1}(x)\right\}, \quad(x \in X)
$$

Then $\mathrm{J}$ is a nonexpansive-type multivalued mapping, but not contractive-type For, if $u_{x}=x-\tan ^{-1}(x) \in J(x)$, for the derivative $u_{x}^{\prime}=1-\frac{1}{1+x^{2}}$, used in the mean value theorem, we have

$$
d\left(u_{x}, u_{y}\right)=\left|u_{x}-u_{y}\right| \leq\left|1-\frac{1}{1+\eta^{2}}\right||x-y|<|x-y|=d(x, y)
$$

where $x<\eta<y$. Clearly $u_{y}=y-\tan ^{-1}(y) \in J(y)$. Similarly, if $u_{x}=\frac{x}{2}-\tan ^{-1}(x) \in J(x)$, we have

$$
\mathrm{d}\left(\mathrm{u}_{\mathrm{x}}, \mathrm{u}_{\mathrm{y}}\right) \leq\left|\frac{1}{2}-\frac{1}{1+\nu^{2}}\right||x-\mathrm{y}|<\mathrm{d}(\mathrm{x}, \mathrm{y}) .
$$

Note that $\eta$ and $\nu$ both depend on $x$ and $\bar{y}$, so $\mathrm{J}$ cannot be a contractive-type mapping. 
Now we prove a fixed point theorem for contractive-type maps on complete metric spaces.

THEOREM 2.3 Let $M$ be a nonempty closed subset of a complete metric space $(X, d)$ and $J: M \rightarrow 2^{M}$ a multivalued contractive-type mapping with closed subsets of $M$ as values. Then there is a point $x \in M$ such that $x \in J(x)$.

PROOF. Let $x_{0}$ be an arbitrary but fixed element of $M$ and choose an $x_{1} \in J\left(x_{0}\right)$ with $d\left(x_{0}, x_{1}\right)>0$. If there is no such $x_{1}$, then $x_{0}$ is already a fixed point of $J$. Since $\mathrm{J}$ is a contractive-type map, there is a $x_{2} \in J\left(x_{1}\right)$ such that

$$
d\left(x_{1}, x_{2}\right) \leq h d\left(x_{0}, x_{1}\right) \text { for some fixed } h, \quad 0 \leq h<1 .
$$

By induction, using the definition of contractive-type map, we obtain a sequence $\left\{x_{n}\right\}$ such that

$$
x_{n+1} \in J\left(x_{n}\right) \text { and } d\left(x_{n}, x_{n+1}\right) \leq h d\left(x_{n-1}, x_{n}\right) \quad(\forall n \geq 1)
$$

This leads to $d\left(x_{n}, x_{n+1}\right) \leq h^{n} d\left(x_{0}, x_{1}\right)$ and for $m>n$, we have

$$
d\left(x_{n}, x_{m}\right) \leq \frac{h^{n}}{1-h} d\left(x_{0}, x_{1}\right)
$$

Since $0 \leq h<1$, we have $d\left(x_{n}, x_{m}\right) \rightarrow 0$ as $m, n \rightarrow \infty$. Thus by the completeness of $X$, we find an element $p \in X$ with $\lim _{n} x_{n}=p$. Since $J(M)=\underset{x \in M}{U} J(x) \subset M$ and $x_{0} \in M$, we have $x_{n} \in M$ for all $n$. Since the sequence $\left\{x_{n}\right\}$ is in the closed set $M$, it follows that $p \in M$. Further, $x_{n} \in J\left(x_{n-1}\right)$ and $J$ being contractive-type implies there is $v_{n} \epsilon$ $\mathrm{J}(\mathrm{p})$ such that

$$
d\left(x_{n}, v_{n}\right) \leq h d\left(x_{n-1}, p\right)
$$

But by using the triangle inequality we have

$$
d\left(p, v_{n}\right) \leq d\left(p, x_{n}\right)+h d\left(x_{n-1}, p\right)
$$

which implies $d\left(p, v_{n}\right) \rightarrow 0$ as $n \rightarrow \infty$. As $v_{n} \in J(p)$ and $J(p)$ is closed, we get $p \in J(p)$, proving that $\mathrm{p}$ is a fixed point of $\mathrm{J}$.

If we take $M=X$ in Theorem 2.3, then we have the following:

COROLLARY 2.4 Each multivalued contractive-type mapping $\mathrm{J}: \mathrm{X} \rightarrow 2^{\mathrm{X}}$ with closed subsets of $\mathrm{X}$ as values, has a fixed point.

This clearly contains the Banach fixed point theorem as a special case. We note that the closedness of $\mathrm{M}$ in Theorem 2.3 is essential.

EXAMPLE 2.5 (a) Let $X=\mathbb{R}$ with the usual metric $d$ and $M=\mathbb{R} \backslash\{0\}$. Define

$$
J(x)=\left\{\frac{x}{2}, \frac{x}{3}, \ldots, \frac{x}{n}\right\} \subset M
$$


for each $x \in M$. Then $J$ is a contractive-type mapping of $M$ with $J(x)$ as a closed subset of $M$ and $h=\frac{1}{2}$. $J$ is a fixed-point-free mapping.

(b) Let $X=\mathbb{R}$ and $M=(0,1]$. Then, the contractive-type closed-valued mapping $\mathrm{J}$ defined in Example 2.1 (c) has no fixed point.

\section{FIXED POINTS OF NONEXPANSIVE-TYPE MAPS ON CERTAIN CONVEX} SUBSETS OF A BANACH SPACE

In this section, we prove fixed point theorems for nonexpansive-type multivalued maps on certain convex bounded subsets of a Banach space. One of these results extends a result due to Husain and Tarafdar [7], contains a Browder's result [2] and generalizes a Kalovitz's result [9].

First we observe that not every nonexpansive-type multivalued mapping on a nonempty closed convex bounded subset $M$ of a Banach space $X$ has a fixed point (cf. [8], Example 2.1). Other such examples might be of interest.

EXAMPLES 3.1 (a) Let $X=c_{0}$, the Banach space of all complex sequences converging to zero with the sup norm: $\left\|\left\{x_{i}\right\}\right\|=\sup \left|x_{i}\right|$. Let $M$ denote the closed unit ball of $c_{0}$. Note that $M$ is not weakly compact because $X$ is not reflexive. For a fixed integer $n_{0} \geq 2$ and for each integer $n \leq n_{0}$, let $f_{n}(x)=\{\underbrace{1, \ldots, 1, x_{1}}_{n-t i m e s}, x_{2}, \ldots\}, x=\left\{x_{n}\right\} \in N^{*}$. Then each $f_{n}$ is a nonexpansive fixed-point-free mapping of $M$ into itself. The compactvalued nonexpansive-type mapping

$$
J(x)=\bigcup_{n=1}^{n_{0}}\left\{f_{n}(x)\right\}
$$

has no fixed point.

(b) Let $\mathrm{X}=\mathrm{C}[0,1]$, the Banach space of continuous real-valued functions on $[0,1]$, which is not reflexive. Let

$$
M=\{f \in X: f(0)=0, f(1)=1,0 \leq f(x) \leq 1\}
$$

$\mathrm{M}$ is closed, convex and bounded. The mapping $\mathrm{J}$

$$
J(f(x))=\left\{x f(x), x^{2} f(x), x^{3} f(x), \ldots\right\}
$$

(where $f \in M$ and $0 \leq x \leq 1$ ) is easily seen to be nonexpansive-type and has no fixed point.

These examples show that we need some stronger conditions either on convex closed and bounded subsets of a Banach space or on the Banach space itself to have a fixed point theorem. In [8] we proved a fixed point theorem (Theorem 2.2) on weakly compact convex subsets under an additional condition (condition $\left({ }^{*}\right)$ ).

Here we prove an improved fixed point theorem for multivalued nonexpansive-type maps on a nonempty convex weakly compact subset of a Banach space $X$ under certain conditions, without $\left(^{*}\right)$ condition. 
We use the following notion due to Opial [14].

A Banach space $X$ is said to satisfy Opial's condition [14] if for each $x \in X$ and each sequence $\left\{x_{n}\right\}$ weakly convergent to $x$,

$$
\underset{n}{\lim \inf }\left\|x_{n}-y\right\|>\underset{n}{\lim \inf }\left\|x_{n}-x\right\|
$$

holds for all $\mathbf{y} \neq \mathbf{x}$.

Every Hilbert space satisfies Opial's condition [14]. Also, if a Banach space $X$ admits a weakly continuous duality mapping [1], then it satisfies Opial's condition [6]. The spaces $l^{p}, 1<\mathrm{p}<\infty$ satisfy Opial's condition [6]. However, there are Banach spaces which fail to satisfy Opial's condition, e.g. $L^{\mathrm{p}}[0,2 \pi](p \neq 2)$ (see [14]).

If the relation $\perp$ is uniformly approximately symmetric [9] in a reflexive Banach space $X$, then $X$ satisfies Opial's condition (see [9]). In a Hilbert space, and more generally for the spaces $\ell^{p}, 1<p<\infty$, the relation 1 is uniformly approximately symmetric. (For further details see [9]).

First, we prove:

PROPOSITION 3.2 Let $M$ be a nonempty closed bounded convex subset of a Banach space $X$. Suppose $J: M \rightarrow 2^{M}$ is a nonexpansive-type compact-valued mapping. Then there exists a sequence $\left\{x_{n}\right\}$ in $M$ and $u_{n} \in J\left(x_{n}\right)$ such that

$$
\left\|x_{n}-u_{n}\right\| \rightarrow 0 \text { as } n \rightarrow \infty \text {. }
$$

PROOF. Consider a sequence of positive numbers $\left\{h_{n}\right\}$ converging to 1 and $0<h_{n}<1$ for all $n \geq 1$ (for instance $h_{n}=\left(1-n^{-1}\right)$ ). For a given point $x_{0}$ of $M$ we define the mapping $J_{n}$ of $M$ into $2^{M}$ by setting

$$
J_{n}(x)=h_{n} J(x)+\left(1-h_{n}\right) x_{0}=\left\{h_{n} u+\left(1-h_{n}\right) x_{0}: u \in J(x)\right\}
$$

The mapping $J_{n}$ does carry $M$ into $2^{M}$, since for each $x \in M, J_{n}(x)$ is the set of convex linear combinations of the points $x_{0}$ and $u \in J(x) \subset M$ and $M$ is convex. Thus we have $J_{n}(M) \subseteq M$. Now we show that for each $n \geq 1, J_{n}$ is a contractive-type mapping such that $J_{n}(x)$ is a closed subset of $M$ for each $x \in M$.

Clearly, for each $x \in M, J_{m}(x)$ being nonempty, if we let $u_{x} \in J_{m}(x)$ then we get

$$
u_{x}=h_{m} v_{x}+\left(1-h_{m}\right) x_{0} \text {, for some } v_{x} \in J(x)
$$

Since $J$ is nonexpansive-type there is a $v_{y} \in J(y)$ for all $y \in M$ such that

$$
\left\|v_{x}-v_{y}\right\| \leq\|x-y\| .
$$

Put $u_{y}=h_{m} v_{y}+\left(1-h_{m}\right) x_{0}$. Clearly, by definition of $J_{m}(y)$ we get $u_{y} \in J_{m}(y)$ and

$$
\left\|u_{x}-u_{y}\right\|=\left\|h_{m}\left(v_{x}-v_{y}\right)\right\| \leq h_{m}\|x-y\|
$$


which proves that $J_{m}$ is contractive-type. Now we show that $J_{m}(x)$ is closed. Put $J_{m}(x)=K$ for fixed $x$ and $m$. Suppose $x_{k} \in K, k=1,2, \ldots$ and $x_{k} \rightarrow y_{0} \in K \subset M$. Since $x_{k} \in J_{m}(x)$, we get

$$
x_{k}=h_{m} u_{k}+\left(1-h_{m}\right) x_{0}, \quad u_{k} \in J(x) .
$$

Using the compactness of $J(x)$, for a convenient subsequence still denoted by $\left\{u_{k}\right\}$ we have $u_{k} \rightarrow u \in J(x)$. Taking the limit as $k \rightarrow \infty$, we get $y_{0}=h_{m} u+\left(1-h_{m}\right) x_{0}$. Thus, by the definition of $J_{m}(x)$ we have $y_{0} \in J_{m}(x)$. This proves that for each $n \geq 1$ and for each $x \in M, J_{n}(x)$ is a closed set. Theorem 2.3 guarantees that for each $n \geq 1, J_{n}$ has a fixed point in $M$, say, $x_{n} \in J_{n}\left(x_{n}\right) \subset M, n=1,2, \ldots$. From the definition of $J_{n}\left(x_{n}\right)$, there is a $u_{n} \in J\left(x_{n}\right)$ such that

Thus

$$
x_{n}=h_{n} u_{n}+\left(1-h_{n}\right) x_{0} .
$$

$$
\left\|x_{n}-u_{n}\right\|=\left\|h_{n} u_{n}+\left(1-h_{n}\right) x_{0}-u_{n}\right\|=\left(1-h_{n}\right)\left\|x_{0}-u_{n}\right\| .
$$

Since $M$ is bounded, $u_{n} \in J\left(x_{n}\right) \subset M$ implies $\left\{\left\|u_{n}-x_{0}\right\|\right\}$ is bounded and so by the fact that $h_{n} \rightarrow 1$ as $n \rightarrow \infty$, we have $\left\|x_{n}-u_{n}\right\| \rightarrow 0$ as $n \rightarrow \infty$.

Proposition 3.3 Let $M$ be a nonempty subset of a Banach space $X$ which satisfies Opial's condition and $\mathrm{J}: \mathrm{M} \rightarrow 2^{\mathrm{M}}$ a compact-valued nonexpansive-type mapping. Let $\left\{x_{n}\right\} \subset M$ be a sequence which converges weakly to an element $x \in M$ and if $y_{n} \in x_{n}-$ $J\left(x_{n}\right)$ such that $\left\{y_{n}\right\}$ converges to $y \in X$ then $y \in x-J(x)$.

PROOF. If $y_{n} \in x_{n}-J\left(x_{n}\right)$, then we can write $y_{n}=x_{n}-u_{n}$ for some $u_{n} \in J\left(x_{n}\right)$. Since $J$ is a nonexpansive-type map, there is a $v_{n} \in J(x)$ such that $\left\|u_{n}-v_{n}\right\| \leq\left\|x_{n}-x\right\|$, it follows that

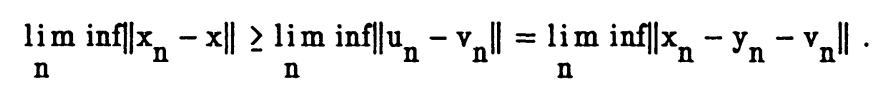

Since every weakly convergent sequence is necessarily bounded, limits in the preceding expression are finite. Now, since $\left\{v_{n}\right\}$ is contained in the compact set $J(x)$, there is a subsequence of $\left\{v_{n}\right\}$, also denoted by $\left\{v_{n}\right\}$, converging to $v \in J(x)$. Therefore,

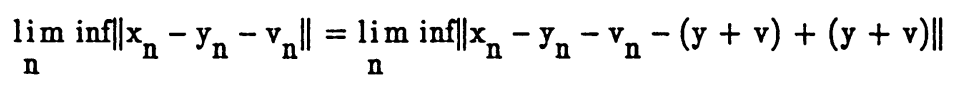

$$
\begin{aligned}
& \underset{n}{\lim \inf \left[\left\|x_{n}-(y+v)\right\|-\left\|\left(y_{n}+v_{n}\right)-(y+v)\right\|\right]} \\
& \underset{n}{\lim \inf \left\|x_{n}-(y+v)\right\|}+\underset{n}{\lim \inf \left(-\left\|y_{n}+v_{n}-y-v\right\|\right)}
\end{aligned}
$$

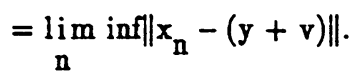

Thus we have shown:

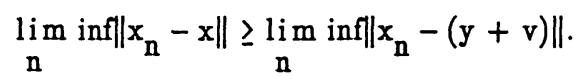


Since $x_{n} \rightarrow x$ weakly, using the Opial's condition we have $x=y+v$, so $y=x-v \in x-J(x)$ and the proposition is proved.

Using Prepositions 3.2 and 3.3, we derive:

THEOREM 3.5 Let $M$ be a nonempty weakly compact convex subset of a Banach space $\mathrm{X}$ which satisfies Opial's condition. Then each nonexpansive-type, compact-valued map $\mathrm{J}: \mathrm{M} \rightarrow 2^{\mathrm{M}}$ has a fixed point.

PROOF. Clearly $M$ is a closed convex and bounded subset of $\mathrm{X}$. By Proposition 3.2 there exists a sequence $\left\{x_{n}\right\}$ in $M$ such that

$$
\left\|y_{n}\right\|=\left\|x_{n}-u_{n}\right\| \rightarrow 0 \text { as } n \rightarrow \infty, \quad u_{n} \in J\left(x_{n}\right)
$$

$M$ being weakly compact, we can find a weakly convergent subsequence $\left\{x_{m}\right\}$ of $\left\{x_{n}\right\}$. Let $x_{0}=w-\underset{m}{-1 i m} x_{m}$. Clearly $x_{0} \in M$ and as above we have $y_{m}=x_{m}-u_{m}\left(u_{m} \in J\left(x_{m}\right)\right)$. Then it follows that $y_{m} \rightarrow 0$ and so by Proposition 3.3 there exists a fixed point $x_{0} \in J\left(x_{0}\right)$.

The following corollaries follow from Theorem 3.4 in view of the remarks given after the definition of Opial's condition.

COROLLARY 3.5 Let $M$ be a nonempty weakly compact convex subset of a Banach space $\mathrm{X}$ having a weakly continuous duality mapping. Then each nonexpansivetype, compact-valued mapping $\mathrm{J}$ of $\mathrm{M}$ into $2^{\mathrm{M}}$ has a fixed point.

COROLLARY 3.6 Let $M$ be a nonempty closed bounded convex subset of a reflexive Banach space (in particular, uniformly convex space) $\mathrm{X}$ which satisfies Opial's condition. Then each nonexpansive-type, compact-valued mapping $\mathrm{J}$ of $\mathrm{M}$ into $2^{\mathrm{M}}$ has a fixed point.

COROLLARY 3.7 Let $M$ be a nonempty closed convex bounded subset of a reflexive Banach space $X$. Suppose the relation 1 is uniformly approximately symmetric, then each nonexpansive-type, compact-valued mapping $\mathrm{J}$ of $\mathrm{M}$ into $2^{\mathrm{M}}$ has a fixed point.

COROLLARY 3.8 Let $M$ be a nonempty closed convex bounded subset of a Hilbert space X. Then each nonexpansive-type, compact-valued mapping $J$ of $M$ into $2^{M}$ has a fixed point.

REMARK 3.9 Corollary 3.8 extends a result due to Husain and Tarafdar [7] and contains a result due to Browder [2] as a special case. Corollary 3.7 generalizes Karlovitz's result [9]. An alternative proof of Theorem 3.4 can be obtained via the result known for nonexpansive maps [12], since closed bounded valued nonexpansive-type maps are nonexpansive. In other words, we obtain a different proof of Theorem 3.2 [12].

Now we give an example which shows that Theorem 3.4 is not true if the Lipschitz constant is greater than 1.

EXAMPLE 3.10 Let $\mathrm{X}=\ell^{2}$, the space of all infinite sequences $x=\left\{x_{i}\right\}_{i \geq 1}$ which are absolutely square-summable with the $\ell^{2}$-norm:

$$
\|x\|=\sqrt{\sum_{i=1}^{\infty}\left|x_{i}\right|^{2}} .
$$

Let $M=\{x \in X:\|x\| \leq 1\}$, which is obviously a closed bounded and convex subset of the reflexive Banach space $\ell^{2}$ and so weakly compact. For a real number $\lambda>1$, we set 


$$
J(x)=\left\{h(1-\|x\|) e_{i}+s_{i}(x): i=1,2, \ldots, n\right\}
$$

for all $x \in M$, where $h<1,0<h \leq\left(\lambda^{2}-1\right)^{1 / 2}, e_{i}=\left\{\delta_{i j}\right\}_{j \geq 1}$ and $s_{i}(x)=$ $\left\{x_{1}, x_{2}, \ldots, x_{i-1}, 0, x_{i}, 0,0, \ldots\right\}$ with 0 in the $i^{\text {th }}$ position.

We see that $J$ is a compact-valued map of $M$ into $2^{M}$. Moreover $J$ is a nonexpansive-type mapping with the Lipschitz constant $\lambda>1$. For, if $u_{x}=h(1-\|x\|) e_{k}+$ $s_{k}(x) \in J(x)$ for some $k$, we put $u_{y}=h(1-\|y\|) e_{k}+s_{k}(y)$. Clearly $u_{y} \in J(y)$ and

$$
\begin{aligned}
\| u_{x}- & u_{y}\left\|^{2}=\right\|\left\{x_{1}-y_{1}, x_{2}-y_{2}, \ldots, x_{k-1}-y_{k-1}, h(\|y\|-\|x\|), x_{k}-y_{k}, \ldots\right\} \|^{2} \\
& =\sum_{j=1}^{\infty}\left|x_{j}-y_{j}\right|^{2}+h^{2}|\|y\|-\|x\||^{2} \\
& \leq\|x-y\|^{2}+h^{2}\|y-x\|^{2}
\end{aligned}
$$

implying

$$
\left\|u_{x}-u_{y}\right\| \leq \sqrt{1+h^{2}}\|x-y\| \leq \lambda\|x-y\|
$$

It is easy to see that $\mathrm{J}$ is a fixed-point-free mapping.

\section{COMMON FIXED POINTS}

There are many interesting common fixed point theorems for a commuting family of maps, e.g. see [3]. Here we prove a common fixed point theorem for a sequence of multivalued contractive-type maps, from which we can derive Theorem 2.3.

THEOREM 4.1 Let $M$ be a nonempty closed subset of a complete metric space $(X, d)$ and $\left\{J_{n}\right\}$ a sequence of closed-valued maps of $M$ into $2^{M}$. Suppose that there exists a constant $h$ with $0 \leq h<1$ such that $\left(^{*}\right)$ : for any two maps $J_{i}$, $J_{j}$ and for any $x \in M, u_{x} \in J_{i}(x)$ implies there is a $u_{y} \in J_{j}(y)$ for all $y$ in $M$ with

$$
d\left(u_{x}, u_{y}\right) \leq h d(x, y)
$$

Then $\left\{J_{n}\right\}$ has a common fixed point.

PROOF. Let $x_{0}$ be an arbitrary element of $M$ and choose $x_{1} \in J_{1}\left(x_{0}\right)$. Then there is $x_{2} \in J_{2}\left(x_{1}\right)$ such that

$$
d\left(x_{1}, x_{2}\right) \leq h d\left(x_{0}, x_{1}\right)
$$

We proceed as in the procf of Theorem 2.3, to show that there is an $x_{n+1} \in J_{n+1}\left(x_{n}\right)$ such that

$$
d\left(x_{n}, x_{n+1}\right) \leq h^{n} d\left(x_{0}, x_{1}\right), \quad(\forall n \geq 1) .
$$

Clearly $\left\{x_{n}\right\}$ is a Cauchy sequence in a complete metric space $X$, and so $p=\lim _{n} x_{n} \in M$. We show that $p$ is a common fixed point of the sequence $\left\{J_{n}\right\}$. such that

Let $J_{m}$ be an arbitrary member of $\left\{J_{n}\right\}$. Since $x_{n} \in J_{n}\left(x_{n-1}\right)$ there is a $u_{n} \in J_{m}(p)$ 


$$
d\left(x_{n}, u_{n}\right) \leq h d\left(x_{n-1}, p\right) .
$$

But then by the triangle inequality (using the last inequality) we have

$$
d\left(p, u_{n}\right) \leq d\left(p, x_{n}\right)+h d\left(x_{n-1}, p\right)
$$

which implies $d\left(p, u_{n}\right) \rightarrow 0$ as $n \rightarrow \infty$. Since $u_{n} \in J_{m}(p)$ and $J_{m}(p)$ is closed, we get $p \in J_{m}(p)$. But then $J_{m}$ being arbitrary, we conclude that $p \in \underset{m \geq 1}{n} J_{m}(p)$.

REMARKS. If we take $\mathrm{J}_{\mathrm{j}}=\mathrm{J}_{1}$ for all $\mathrm{j} \geq 1$ in Theorem 4.1 , then again we obtain Theorem 2.3. Further, any pair of contraction maps having the same Lipschitz constant and satisfying $(*)$ have a common fixed point.

ACKNOWLEDGEMENT. This work was partially supported by an NSERC grant.

\section{References}

1. Browder, F.E.; On a Theorem of Beurling and Livingston; Canadian J. Math., 17(1965), 367-372.

2. Browder, F.E.; Fixed Point Theorems for Non-Compact Mappings in Hilbert Space; Proc. Nat. Acad. Sci. U.S.A., 53(1965); 1272-1276.

3. Browder, F.E.; Non-Expansive Non-Linear Operators in a Banach Space; Proc. Nat. Acad. Sci. U.S.A., 54(1965), 1041-1044.

4. Edelstein, M.; An extension of Banach's Contraction Principle; Proc. Amer. Math. Soc., 12(1961), 7-10.

5. Edelstein, M.; On Fixed and Periodic Points Under Contractive Mappings; Journal London Math. Soc., 37(1962), 74-79.

6. Gossez, J.P. and Lami Dozo, E.; Some Geometric Properties Related to the Fixed Point Theory for Non-Expansive Mappings; Pacific. J. Math.. 40 (1972) $565-573$.

7. Husain, T. and Tarafdar, E.; Fixed Point Theorems for Multi-Valued Mappings of Non-Expansive Type; Yokohama Math. J., 28(1980), 1-6.

8. Husain, T. and Latif, A.; "Fixed Points of Multivalued Nonexpansive Maps"; Math. Japonica 33 (1988), 385-391.

9. Karlovitz, L.A.; On Non-Expansive Mapping; Proc. Amer. Math. Soc., 55(1976), $321-325$.

10. Kasahara, S.; On Some Generalizations of the Banach Contraction Theorem; Publ. RISM Kyoto Univ., 12(1976), 427-437

11. Kirk, W.A.; A Fixed Point Theorem for Non-Expansive Mappings which do not Increase Distance; Amer. Math. Monthly, 72(1965), 1004-1006.

12. Lami Dozo, L.; "Multivalued Nonexpansive Mappings and Opial's Condition", Proc. Amer. Math. Soc., 38(1973), 285-292.

13. Nadler, S.B.; Multi-Valued Contraction Mappings: Pacific J. Math., 30(1969), 475-487.

14. Opial, Z.; Weak Convergence of the Sequence of Successive Approximations for NonExpansive Mappings; Bull. Amer. Math. Soc., 73(1967), 591-597.

15. Rakotch, E.; A Note on Contraction Mappings; Proc. Amer. Math. Soc., 13(1962), $459-462$.

16. Rakotch, E.; A Note on Locally Contractive Mappings; Bull. Res. Council Israel, 40(1962), 188-191. 


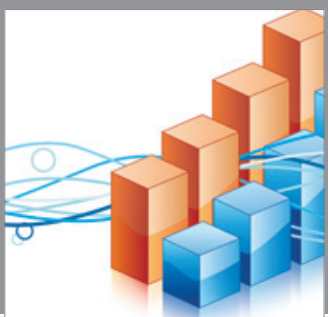

Advances in

Operations Research

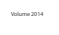

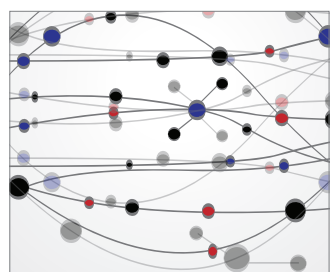

\section{The Scientific} World Journal
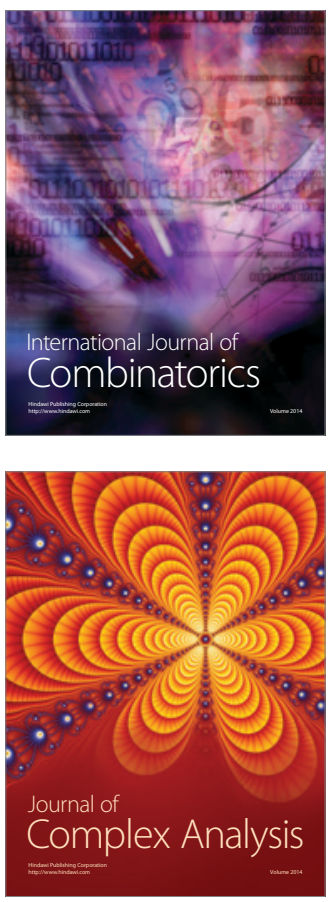

International Journal of

Mathematics and

Mathematical

Sciences
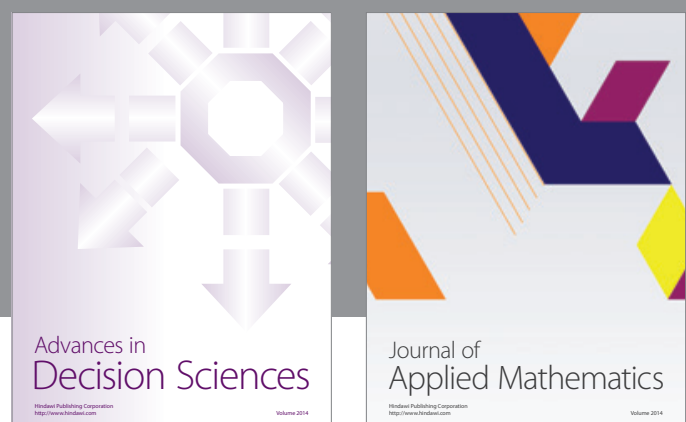

Journal of

Applied Mathematics
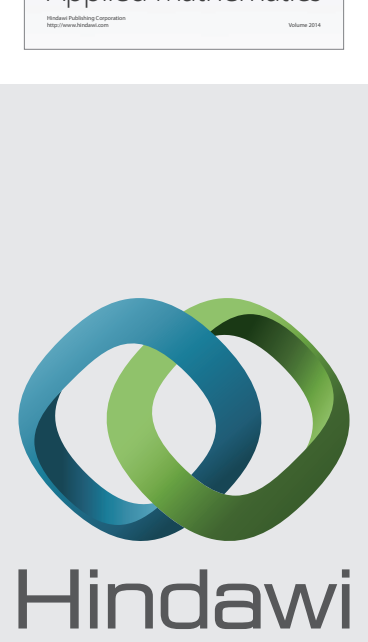

Submit your manuscripts at http://www.hindawi.com
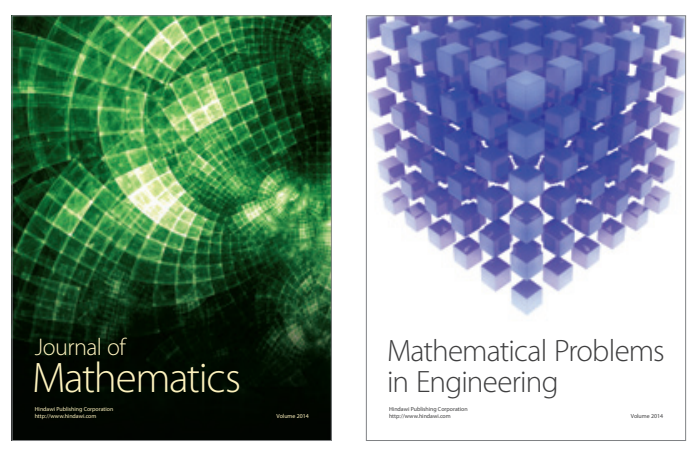

Mathematical Problems in Engineering
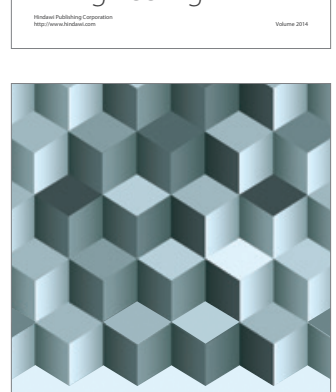

Journal of

Function Spaces
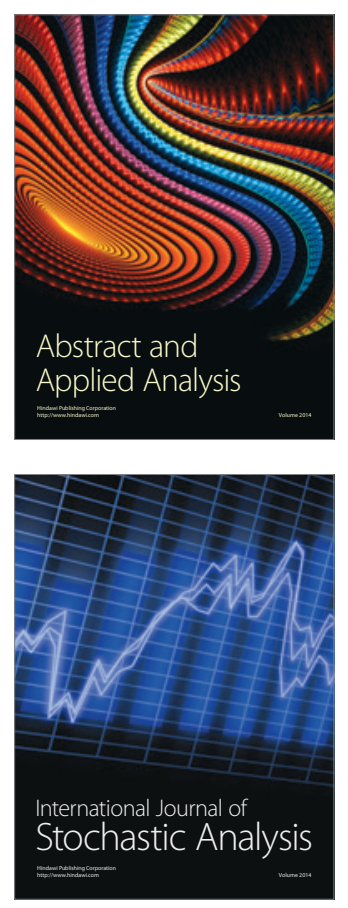

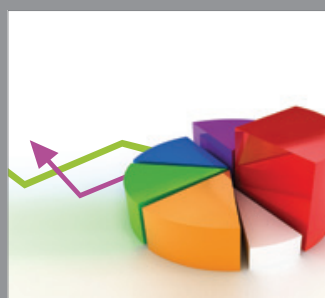

ournal of

Probability and Statistics

Promensencen
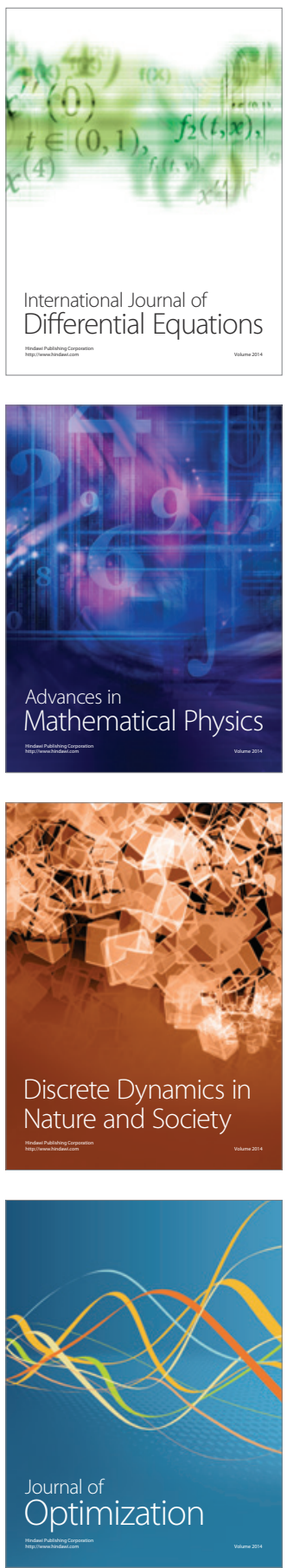